\title{
Einbindung durch Anerkennung? Nichtstaatliche Gewaltakteure und das humanitäre Völkerrecht
}

\author{
Stefanie Herr*
}

\begin{abstract}
The protection of civilians in armed conflict requires that not only states but also non-state armed groups comply with international humanitarian law. This article investigates the conditions under which armed groups commit themselves to humanitarian norms. For this purpose, the commitment of one armed group (the Sudan People's Liberation Movement/Army) to the ban on antipersonnel mines is investigated. The analysis shows that the actor's quest for recognition by the international community played a crucial role in its decision to accept humanitarian standards. Results are based on expert interviews conducted in Kenya and South Sudan.
\end{abstract}

Keywords: Non-state armed groups, international humanitarian law, South Sudan, recognition Nichtstaatliche Gewaltakteure, Humanitäres Völkerrecht, Südsudan, Anerkennung

$\mathrm{D}$ ie Mehrheit bewaffneter Konflikte findet heutzutage nicht mehr zwischen, sondern innerhalb von Staaten statt. An solchen innerstaatlichen Gewaltkonflikten ist mindestens ein nichtstaatlicher Gewaltakteur (non-state armed group, NSAG) ${ }^{1}$ beteiligt. Opfer dieses Trends sind in erster Linie Zivilisten: Die Liste an Gräueltaten reicht dabei vom Einsatz nicht-diskriminierender Waffen (wie Streubomben oder Antipersonenminen) über Massenvergewaltigungen bis hin zur Zwangsrekrutierung von Kindersoldaten. UNGeneralsekretär Ban Ki Moon hebt daher hervor, eine der wichtigsten Herausforderungen des 21. Jahrhunderts sei es, die Gewalt gegen Zivilisten in innerstaatlichen Konflikten einzuschränken (UN 2009).

Die Arbeit verschiedener nichtstaatlicher Organisationen wie des Internationalen Komitees des Roten Kreuzes oder des Centre for Humanitarian Dialogue und deren Praxis der Inklusion von NSAGs hat gezeigt, dass es eine Alternative zur Dämonisierung und Kriminalisierung dieser Akteure gibt (Hofmann/Schneckener 2010). Dieser Artikel widmet sich daher der Frage, wie nichtstaatliche Gewaltakteure von der Notwendigkeit der Beachtung humanitärer Standards überzeugt werden können. Ich argumentiere, dass nichtstaatliche Gewaltakteure dann zur Einhaltung humanitärer Standards bereit sind, wenn sie nach (politischer) Anerkennung durch die internationale Gemeinschaft streben. Theoretische Vorannahmen legen nahe, dass der Wunsch nach Anerkennung bei der Einhaltung bestimmter Normen eine zentrale Rolle spielt. An einem konkreten Beispiel - dem Verzicht auf Antipersonenminen durch die Sudan People's Liberation Movement/Army (SPLM/A) im Südsudan - verifiziere ich mein theoretisches Argument. Die Analyse basiert auf Experteninterviews, die die Autorin im Rahmen von Feldforschung in Kenia und dem (Süd-) Sudan durchführte. ${ }^{2}$

* Stefanie Herr ist wissenschaftliche Mitarbeiterin an der Hessischen Stiftung Friedens- und Konfliktforschung, Frankfurt am Main. Dieser Beitrag ist double-blind peer-reviewed.

1 Eine NSAG wird als „, any armed actor with a basic structure of command operating outside state control that uses force to achieve its political or allegedly political objectives" verstanden (Geneva Call 2005: 10).

2 Insgesamt wurden 29 Interviews durchgeführt. Als Experten galten dabei jene Personen, die a) Verantwortung für eine Problemlösung tragen, oder b) über den privilegierten Zugang zu Informationen über Entscheidungsprozesse verfügen beziehungsweise c) selbst Teil des Handlungsfeldes sind, das den Forschungsgegenstand ausmacht (Meuser/Nagel 1991: 443). Dazu zählten in meinem Fall sowohl ehemalige Kämpfer und Kindersoldaten der NSAG, als auch NGO-Mitarbeiter, Journalisten und Wissenschaftler. Eine detaillierte Liste der geführten Interviews ist bei der Autorin einsehbar.

\section{Nichtstaatliche Gewaltakteure und das Humanitäre Völkerrecht}

In innerstaatlichen Konflikten stellt das Humanitäre Völkerrecht den vorherrschenden normativen Rahmen dar, an dem sich NSAGs messen lassen müssen. Im Vergleich mit Vereinbarungen, die bei zwischenstaatlichen Konflikten greifen, existieren jedoch weit weniger völkerrechtliche Normen für nicht-internationale Konflikte (Sassòli 2006). Zudem zielen diese häufig nur auf die Zustimmung von staatlichen Akteuren $\mathrm{ab}$ und erklären die Anerkennung durch NSAGs für irrelevant. Dieser Missstand ist vor allem auf die Tatsache zurückzuführen, dass Staaten oft fürchten, eine auch nur partielle Völkerrechtsubjektivität von NSAGs könnte einen ungewollten Legitimationsprozess einleiten (Bassiouni 2009).

Damit verkennen sie aber die Realität. Dass nichtstaatliche Gewaltakteure an humanitäre Normen gebunden werden können, zeigt das Engagement von Geneva Call. Die Genfer NGO nimmt bei der Förderung der humanitären Verantwortung nichtstaatlicher Gewaltgruppen eine Vorreiterrolle ein. Sie bemüht sich seit dem Jahr 2000 weltweit, NSAGs von der Notwendigkeit der Beachtung humanitärer Standards zu überzeugen, und setzt dies konkret mit dem Verbot von Antipersonenminen (APMs), dem Kampf gegen den Einsatz von Kindersoldaten und gegen sexuelle Gewalt in Kriegshandlungen um. Den Anfang nahm das Engagement der NGO mit dem Minenverbot: Geneva Call entwickelte ein Deed of Commitment for Adherence to a Total Ban on Anti-Personnel Mines (im Folgenden Deed of Commitment, DoC), mit dessen Unterzeichnung sich bewaffnete Gruppen dazu verpflichten, auf den Einsatz, die Produktion, die Lagerung und den Weiterverkauf von Antipersonenminen zu verzichten. Seit ihrer Gründung stand Geneva Call mit mehr als 60 NSAGs im Dialog. Bis zum Jahr 2012 haben 42 Gruppen das DoC unterzeichnet. Inzwischen existieren ähnliche Verzichtserklärungen auch zur Vermeidung sexueller Gewalt in Kriegshandlungen sowie zum Schutz von Kindern vor den Auswirkungen bewaffneter Konflikte. Neu an dem Engagement der Genfer NGO ist zweierlei: Zum einen tritt Geneva Call nicht gegenüber Staaten oder der Privatwirtschaft als Normunternehmer auf, sondern richtet ihr Engagement explizit auf nichtstaatliche Gewaltakteure. Zum anderen stellt die NGO mit dem Deed of Commitment einen 
innovativen Mechanismus bereit, mit dessen Hilfe sich NSAGs an völkerrechtliche Normen binden können und erstmals Unterzeichner eines international respektierten Regelwerks werden. Lange Zeit besaßen NSAGs nur die Möglichkeit, eine völkerrechtliche Norm durch die Herausgabe einer unilateralen Willenserklärung oder durch die Integration in eigene Verhaltenskodizes anzuerkennen - solche Bekenntnisse fanden allerdings in der Öffentlichkeit wenig Beachtung (IKRK 2007).

Unklar ist allerdings noch, was NSAGs zu einem solchen bindenden Bekenntnis zu humanitären Normen bewegt. Zwar richtet sich die wissenschaftliche Aufmerksamkeit in den letzten Jahren verstärkt auf nichtstaatliche Akteurskonstellationen, die Frage allerdings, wann NSAGs bereit sind, sich an humanitäre Normen zu binden, bleibt dabei unbeantwortet. Diese Frage ist deshalb besonders relevant, weil ein öffentliches Bekenntnis zu humanitären Normen ein wichtiger Schritt zur besseren Durchsetzbarkeit Humanitären Völkerrechts ist. Clapham hebt hervor: „Turning to rebels, theories concerning why they should comply with these norms are of more than academic interest" (2006: 511).

In der völkerrechtlichen Debatte finden sich erste Anzeichen dafür, dass (politische) Anerkennung durch die internationale Gemeinschaft für NSAG einen wichtigen Anreiz bei der Einhaltung völkerrechtlicher Normen darstellen kann. Die Ablehnung von Staaten, nicht-internationale Konflikte als solche zu deklarieren und NSAGs als Völkerrechtssubjekte anzuerkennen, verspielt gleichzeitig eine wichtige Chance, diese an humanitäre Normen zu binden, so die Meinung in der Literatur. Bassiouni betont: "Since governments refuse to give belligerent and insurgent groups international legal recognition, the latter may seek to acquire such standing by declaring themselves willing to abide by IHL. [Thereby], they may seek partial recognition before the international community" (Bassiouni 2008: 737). Der Wunsch nach Anerkennung dient dann als Anreiz Normverletzungen zu vermeiden und zu zeigen, dass NSAGs - ähnlich wie Staaten - Verantwortung für ihr Handeln übernehmen können. Achtung des Humanitären Völkerrechts „,is an important way for armed opposition groups to achieve some standing. The more they comply with IHL, the more they will gain recognition" (Henckaerts 2003: 133). Der Wunsch nach Anerkennung einer NSAG gilt somit in der völkerrechtlichen Debatte als wichtiger Erklärungsfaktor für die Normakzeptanz einer NSAG. Gleichzeitig wurde der Wunsch nach Anerkennung einer NSAG weder theoretisch begründet und an bestehende Arbeiten in den internationalen Beziehungen geknüpft, noch einer systematischen empirischen Analyse unterzogen. Diese Forschungslücke gilt es im Folgenden zu schließen. ${ }^{3}$

\section{Der Wunsch nach Anerkennung: Ein wichtiger Anreiz für NSAGs}

Seit einigen Jahren wird das Konzept der Anerkennung und dessen Einfluss auf das Verhalten von staatlichen Akteuren in

3 Vergleiche zu einer detaillierten Analyse alternativer Erklärungsmöglichkeiten der Normakzeptanz durch die SPLM/A Herr (2010). den Internationalen Beziehungen vermehrt diskutiert. Unter Anerkennung wird dabei ein sozialer Akt verstanden, durch den ein Akteur als Partner im sozialen Dialog akzeptiert und somit als Subjekt mit legitimem sozialem Ansehen konstituiert wird (Lindemann 2011). Zwar sind diese Ansätze der IBForschung noch sehr staatszentriert, sie geben jedoch umso mehr Anlass zur Vermutung, dass sich auch nichtstaatliche Gewaltakteure durch den Wunsch nach Anerkennung in ihrem Verhalten beeinflussen lassen: Wenn Anerkennung bei staatlichen Akteuren - die bereits Mitglieder der internationalen Gemeinschaft sind - eine so große Rolle spielt, dann dürfte sie für NSAGs, die in der Regel nur als outlaws gelten, erst Recht von Bedeutung sein.

Es kann mehrere Gründe dafür geben, dass kollektive Akteure, wie NSAGs, nach Anerkennung streben - emotionale und instrumentelle. Zunächst zu ersteren: Für Anerkennungstheoretiker werden Identitäten intersubjektiv bestimmt. Wer oder was ein Akteur ist, ist das Resultat vieler sich überschneidender Aktionen und Reaktionen - eine Wechselbeziehung zwischen der Selbst- und Fremdwahrnehmung eines Akteurs. Der Wunsch nach Anerkennung resultiert aus der Entschlossenheit eines Akteurs das eigene Selbstverständnis vorzubringen. Wenn die Identität einer NSAG nicht anerkannt wird, so Murray (2012: 135), „it suffers disrespect because in being denied membership in the collective, the recognition seeking [actor] can be represented as 'illegitimate' or 'second-rate'". Akteure versuchen eine solche öffentliche Demütigung und Verunglimpfung in der Regel zu vermeiden. Stattdessen streben sie danach, Teil einer internationalen Wertegemeinschaft zu sein.

Auch instrumentelle Gründe können für den Wunsch nach Anerkennung eine wichtige Rolle spielen. Wird NSAGs Anerkennung verweigert, müssen sie um ihre Existenz fürchten, dann nämlich, wenn diese Missachtung auch Auswirkungen auf die Beschaffung materieller Ressourcen hat oder die internationale Gemeinschaft die militärische Zerschlagung der NSAG duldet. Für nichtstaatliche Gewaltakteure scheint daher bereits eine „schwache" Form der Anerkennung erstrebenswert, die darüber entscheidet, ob ein Akteur überhaupt das Recht besitzt zu existieren (Alan/Keller 2011). Während eine solche minimalistische Anerkennungsform zwischen Staaten als selbstverständlich gelten kann, ist die Akzeptanz der Existenz eines nichtstaatlichen Gewaltakteurs für diese schon eine Besonderheit. Oft streben NSAGs genau danach, dass ihre Existenz nicht in Frage gestellt wird.

Nichtstaatliche Gewaltakteure können aber noch aus einem dritten Grund den Wunsch nach Anerkennung haben. Akteure rechnen nicht nur materielle Kosten und Nutzen auf, sondern beziehen auch symbolische Kosten in ihre Rechnung mit ein: Anerkennung geht in der Regel Hand in Hand mit Status- und Reputationsgewinnen. Ein anerkannter Akteur, der eine gewisse Reputation besitzt, hat es leichter, mit anderen in Verhandlungen zu treten und sich auf dem politischen Parkett zu bewegen. Anerkennung kann sogar Vertrauen schaffen: Wird ein Akteur X durch bestimmte Mitglieder der internationalen Gemeinschaft als Gesprächspartner anerkannt, können andere diesem Beispiel folgen. Fehlende Anerkennung hingegen bringt bestimmte Kosten mit sich, 
wie etwa eine geringere Glaubwürdigkeit. Lindemann betont: „In an asymmetrical social conflict, the weaker party can compensate for its material inferiority through better moral standing" (Lindemann 2011: 70). Dieser Fakt ist insbesondere dann für NSAGs von Bedeutung, wenn diese eine Regierungsbeteiligung oder die Machtübernahme in einem eigenen Staat anstreben, also ein „shadow of future statehood" existiert (Börzel/Risse i.E.). In solchen Fällen riskieren NSAGs, mit Verstößen gegen das Völkerrecht ihre Unterstützung und Einbindung sowohl in der eigenen Gesellschaft als auch international aufs Spiel zu setzen.

Dieser Artikel geht demnach von folgender Annahme aus: Haben nichtstaatliche Gewaltakteure den Wunsch, von der internationalen Gemeinschaft anerkannt zu werden, und ist diese zumindest gewillt, ihnen dies zu gewähren, bekennen sich NSAGs im Gegenzug auch zu den von der internationalen Gemeinschaft geteilten Normen und Werten, allen voran dem humanitären Völkerrecht.

\section{Lehren aus dem Südsudan}

Mein Argument belege ich im Folgenden durch die Analyse eines Falls. Hierzu untersuche ich die Normakzeptanz - in diesem Fall konkret das Verbot von Antipersonenminen einer NSAG, der Sudan People's Liberation Movement/Army (SPLM/A) im Südsudan. Die Analyse dieses Falls ist besonders relevant, weil die SPLM/A a) vor dem Verbot von Antipersonenminen nachweislich Minen einsetzte, und sie sich b) zu diesem Zeitpunkt weiterhin in einem gewaltsamen Konflikt befand. Der Verzicht auf einen Landmineneinsatz war folglich "unbequem". 4

Der sudanesische Bürgerkrieg zwischen der Sudan Peoples Liberation Movement/Army (SPLM/A) und nördlichen Regierungstruppen gilt als einer der längsten bewaffneten Konflikte der Gegenwart. Seit der Unabhängigkeit des Landes im Jahr 1956 bis zur Unterzeichnung des umfassenden Friedensabkommens zwischen Norden und Süden im Juni 2005 kannte das Land nur ein Jahrzehnt Frieden. Der erste Bürgerkrieg fand mit dem Addis-Abeba-Abkommen im Februar 1972 ein vorläufiges Ende. Nachdem es der Regierung allerdings nicht gelungen war, wirtschaftliche und politische Ungleichheiten zwischen dem Norden und Süden des Landes auszugleichen, brach 1983 erneut ein Bürgerkrieg aus. Meuternde Armeeangehörige flüchteten aus dem Süden nach Äthiopien und gründeten die SPLM/A. John Garang übernahm das Kommando über die heterogenen Truppen. Die militärischen Auseinandersetzungen, verstärkt durch ethnische und militärische Spaltungen innerhalb des Südens, erfassten beinahe das ganze Land. Sie fanden erst 2005 nach langjährigen Friedensverhandlungen ein Ende. Dennoch bestehen auch nach der Unabhängigkeit des Südsudans im Juni 2011 Spannungen zwischen dem Norden und dem Süden.

4 Neben diesen Kriterien spielten auch pragmatische Überlegungen bei der Fallauswahl eine wichtige Rolle: Da über nichtstaatliche Gewaltakteure in der Regel sehr wenige Informationen nach außen dringen, wurde jene Gruppierung ausgewählt, bei der der Zugang zu Informationen gesichert war und die Sicherheitslage vor Ort Feldforschung möglich machte.

\subsection{Zur Normakzeptanz einer NSAG: Das Bekenntnis der SPLM/A zum Verbot von Antipersonenminen}

Der Südsudan gilt als eines der am stärksten von Landminen betroffenen Gebiete weltweit. Ein Drittel des Landes ist mit Minen kontaminiert. Während des Bürgerkriegs setzten sowohl Regierungstruppen und die SPLM/A als auch rivalisierende NSAGs im Süden Antipersonenminen ein (Landmine Monitor 1999). Antipersonenminen sind für NSAGs von großer strategischer Bedeutung: Sie sind billig, leicht zu verlegen, können im Bedarfsfall sogar selbst hergestellt werden und haben eine große Wirkung. Da bewaffnete Gruppen geringere militärische Ressourcen besitzen als Staaten, sind sie mit größerer Wahrscheinlichkeit auf die Nutzung von AP-Minen angewiesen (Interview \#3).

Trotzdem stieg innerhalb der SPLM/A Mitte der 1990er Jahre langsam das Bewusstsein für die negativen Effekte von APMinen. Bei einer Konferenz im Südsudan, an der wichtige Vertreter der SPLM/A, internationale und lokale NGOs sowie zivilgesellschaftliche Gruppen teilnahmen, fanden erste Diskussionen über den Einsatz von Antipersonenminen statt (Interview \#5). Sowohl die SPLM/A als auch die sudanesische Regierung baten um internationale Unterstützung bei der Beseitigung von Minen. Mit einer Starthilfe externer NGOs beauftragte die SPLM/A 1997 die NGO Operation Save Innocent Lives (OSIL) in den von der SPLM/A kontrollierten Gebieten mit der Minenräumung. Im Süden wurden zudem mit Hilfe verschiedener humanitärer Organisationen Aufklärungsmaßnahmen über die Risiken von APMs durchgeführt. Dennoch verlegte die NSAG auch in dieser Zeit noch Minen (Interview \#3). ${ }^{5}$

Im März 2000 nahmen zudem Vertreter der SPLM/A an einer Konferenz über den Einsatz von Minen mit anderen nichtstaatlichen Akteuren in Genf teil, die von der Swiss Campaign to Ban Landmines veranstaltet wurde. Zum Abschluss der Konferenz bekannten sich Vertreter der SPLM/A zum ersten Mal mündlich zu einem Minenverbot (Interview \#6). Eineinhalb Jahre später, im Oktober 2001, reiste schließlich ein Vertreter der SPLM/A, Nhial Dheng Nhial, nach Genf um das Deed of Commitment zu unterzeichnen. Zwar beschuldigte der sudanesische Staat die SPLM/A auch nach dieser Unterzeichnung, weiterhin Minen einzusetzen (Landmine Monitor 2001). Diese Berichte wurden jedoch nie unabhängig bestätigt. Zudem zeigten Interviews mit Vertretern der im Südsudan ansässigen NGOs, dass diese Anschuldigungen haltlos waren (Interview \#5). Der Normakzeptanz folgte somit auch eine Normeinhaltung. Umso relevanter ist die Frage, wie ein solcher Wandel in der Minenpolitik der NSAG zu erklären ist.

\subsection{Der Wunsch nach Anerkennung als Erklärungsfaktor für die Akzeptanz einer Norm}

Wie oben herausgearbeitet, ist der Wunsch nach Anerkennung einer NSAG dann besonders groß, wenn die Gruppe po-

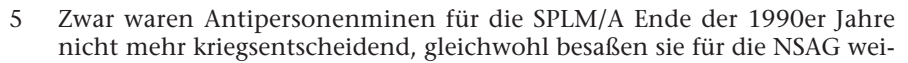
nicht mehr kriegsentscheidend, gleichwohl besaßen sie für
terhin eine wichtige strategische Bedeutung (Interview \#7). 
litische Ziele verfolgt und den bewaffneten Konflikt nicht nur zur Selbstbereicherung fortführtt. Die SPLM/A kämpfte von Beginn des Krieges an gegen strukturelle Ungleichheiten im ganzen Land (Johnson 1998). John Garang forderte die Umwandlung des Sudan in eine säkulare, dezentrale Demokratie, in der Nord- und Südsudanesen gleiche Rechte zugestanden werden. Das Streben nach Selbstbestimmung stand daher im Mittelpunkt der Agenda (Interview \#16). Die SPLM/A verfolgte mit ihrem Kampf somit klare politische Ziele: Der Einsatz von Gewalt diente der Veränderung des Status quo. Zur Erreichung ihrer Ziele war sie folglich auf eine gute Reputation angewiesen - spätestens ab dem Zeitpunkt, als sie sich eine reale Chance ausrechnete, selbst die Kontrolle über den Staat zu übernehmen, und der von Börzel und Risse postulierte „shadow of future statehood" einsetzte. „The WAR could be fought with Kalashnikovs, but a future peace had to be won in a different manner", betont Rolandsen (2005: 129, Hervorheb. im Orig.). Wie wichtig der SPLM/A dabei die Anerkennung durch die international Gemeinschaft war, betonte auch ein Interviewpartner: "They wanted the international community to respect them. They were actually starting to behave quite well, but the international community was lagging behind. I remember talking to people, the British government and the German government and other officials who would still say, [...] they are just rebels and they wanted to get over that thing that they were just rebels. They felt that they are a responsible liberation movement" (Interview \#16).

Der Wunsch nach Anerkennung der SPLM/A wurde auch durch die Tatsache verstärkt, dass sich Mitte der 1990er Jahre die Aufmerksamkeit sowohl der vor Ort tätigen NGOs als auch der UN auf den Einsatz von Antipersonenminen durch die SPLM/A erhöhte. Bereits 1995 wurde eine Frauenrechtskonferenz in Nairobi, mitorganisiert von der Kenyan Coalition to Ban Landmines (KCAL), als Plattform genutzt, um den Umgang der SPLM/A mit Antipersonenminen anzuprangern. Der Kenyan Coalition to Ban Landmines kam auch in der Folgezeit eine wichtige Rolle zu: Immer wieder lenkte sie die Aufmerksamkeit der Öffentlichkeit auf den Mineneinsatz der SPLM/A (Interview \#5). Ende der 1990er Jahre wurden zudem gleich zwei ausführliche Berichte veröffentlicht, die sich mit der Landminenproblematik im Südsudan beschäftigten und den Mineneinsatz der SPLM/A stark kritisierten (Landmine Monitor 1999): Sowohl das UN Department of Humanitarian Affairs als auch Human Rights Watch forderten die SPLM/A auf, auf den Einsatz von Antipersonenminen zu verzichten. Minen waren auch Gesprächsthema in einem Treffen zwischen der SPLM/A und dem Sonderbeauftragten der VN Ende der 1990er Jahre. Sowohl innerstaatliche als auch internationale NGOs griffen in der Folge den Einsatz von Antipersonenminen durch die SPLM/A auf und verurteilten ihn öffentlich (Interview \#7).

Die SPLM/A war sich der Erwartungen, die auf ihr lasteten, durchaus bewusst. Das hob der SPLM/A-Repräsentant Nhial Deng Nhial bei der Unterzeichnung des DoC hervor: „The SPLM/A is fully conscious of the fact that although it is a nonstate actor, the world has come to expect from it humanitarian standards, approaching if not on a par with those expected of sovereign governments" (SPLM/A 2001). Die Unterzeichnung des Deed of Commitments bot dabei ein willkommenes
Instrument, mit dessen Hilfe die SPLM/A zeigen konnte, dass auch eine nichtstaatliche bewaffnete Gruppe Verantwortung für ihr Handeln übernehmen kann. Zwar hatte die NSAG bereits Anfang der 1990er Jahre im Zuge der von den VN koordinierten Operation Lifeline Sudan (OLS) die Möglichkeit, sich auf internationaler Ebene zu profilieren. Nachdem sich die Reputation der SPLM/A Ende der 1990er jedoch wieder verschlechtert hatte, ${ }^{6}$ nahm die SPLM/A die Gelegenheit wahr, diese durch die Unterzeichnung des Deed of Commitment zu verbessern. Ein Mitarbeiter Geneva Calls kommentierte diesen Schritt in einem Interview folgendermaßen: "They were hoping [to achieve] some political gains [...] they could appear as a movement that is abiding with international law, that would enhance their reputation, as a movement that is sensitive to international human rights standard" (Interview \#6).

Die Analyse zeigt zudem, dass auch dem festlichen Akt der Unterzeichnung der Verzichtserklärung dabei eine wichtige Rolle zukommt: Für diesen Anlass wurden Vertreter der NSAG nach Genf eingeladen und trafen nicht nur auf Mitarbeiter von Geneva Call, sondern wurden auch offiziell von Repräsentanten des Genfer Kantons begrüßt. Die Unterzeichnung fand anschließend im geschichtsträchtigen Alabama-Saal des Rathauses statt, in dem 1864 die Genfer Konventionen unterzeichnet worden waren (Interview \#1). Der Besuch der SPLM/A in Genf war folglich eine der wenigen Möglichkeiten, in denen die NSAG sich auf internationalem Parkett bewegen und ihr Bekenntnis zum Verbot von Antipersonenminen global bekannt machen konnte (Interview \#16). Dies wird auch in der Aussage von Nhial Deng Nhial deutlich, der bei der Unterzeichnung des DoC hervorhob: „I am very thankful to both the Swiss and Geneva governments and people for their brave understanding by allowing us, we the non-state actors, to have such an international forum in which to interact" (SPLM/A 2001). Zwar geben solche Äußerungen der NSAG nur bedingt Aufschluss über die Motivation der Gruppe. Dass der Wunsch nach Anerkennung bei der Entscheidung der SPLM/A auf Landminen zu verzichten eine wichtige Rolle gespielt hat, wurde jedoch auch von anderen Experten bestätigt. Ein Interviewpartner, der in besonderem Maße mit der Minenpolitik der Gruppe vertraut war, betont: "I believe that the SPLA's motives were predominantly related to the opportunities presented by collaborating with GC [Geneva Call] as a formallyrecognised NSA [non-state actor]" (Interview \#7).

Zudem spielte für das Verbot von Minen eine wichtige Rolle, dass die sudanesische Regierung die Ottawa-Konvention noch nicht unterzeichnet hatte und die SPLM/A sich so besser, sprich verantwortungsvoller, als der Konfliktgegner zeigen konnte: „[...] it was a way to appear better than the opponent government", so ein Mitarbeiter von Geneva Call (Interview \#6). Der SPLM/A war es demnach wichtig zu beweisen, dass sie sich an internationale Normen hält und in der Lage ist, nach Ende des Krieges Verantwortung für die sudanesische Bevölkerung zu übernehmen. Mit Erfolg: Die sudanesische Regierung sah sich

6 Diskussionen entfachte insbesondere die Tatsache, dass die SPLM/A von allen im Süden des Landes tätigen NGOs die Unterzeichnung eines Memorandum of Understanding verlangte, das bestimmte Kriterien für die Durchführung humanitärer Arbeit vorsah und insbesondere von NGOs forderte, in Einklang mit den Zielen des humanitären Flügels der SPLM/A zu arbeiten (vgl Riehl 2001). 
durch den Verzicht der NSAG so unter Zugzwang gesetzt, dass sie 2003 ihrerseits die Ottawa-Konvention unterzeichnete.

\section{Fazit}

Die Analyse zeigt, dass NSAGs durch ein öffentliches Bekenntnis zu humanitären Normen nach Anerkennung durch die internationale Gemeinschaft streben. Dies hat in der Praxis zur Folge, dass nichtstaatliche Akteure im Umgang mit NSAGs Staaten in der Regel überlegen sind: Letztere scheuen sich, direkt mit NSAGs in Dialog zu treten, aus Angst die Legitimität einer bewaffneten Gruppe zu erhöhen. Für NGOs hingegen stellen sich Fragen von staatlicher Souveränität und NichtEinmischung nicht im gleichen Maße. Eine Möglichkeit für staatliche Akteure bietet sich daher in der Indienstnahme von NGOs.

Dies zeigt auch das hier untersuchte Engagement der NGO Geneva Call. Sie bietet NSAGs die Gelegenheit, ihr Engagement öffentlich bekannt zu machen und somit Verantwortung für ihr Verhalten zu übernehmen. Vertreter verschiedener NSAGs nahmen bereits an einer Vielzahl von Konferenzen in Genf teil, bei denen sie die Möglichkeit hatten, auf Augenhöhe mit Staatsvertretern zu diskutieren. Durch die Teilnahme an solchen Konferenzen werden NSAGs als Gesprächspartner akzeptiert und ihrer wichtigen Rolle für Friedensprozesse Rechnung getragen. Nichtstaatliche Gewaltakteure realisieren folglich, dass sie - bei Akzeptanz und Einhaltung humanitärer Normen - Teil einer globalen Bewegung werden. Gleichzeitig wird durch die öffentliche Aufmerksamkeit, die diesen Treffen zuteilwird, der Respekt für festgesetzte Vereinbarungen auch langfristig erhöht: NSAGs, die sich einmal öffentlich zu humanitären Normen bekannt haben, werden in den meisten Fällen auch versuchen, diese durchzusetzen - alleine schon um ihre neu gewonnene Reputation nicht wieder aufs Spiel zu setzen (Sassòli 2006).

Der Wunsch nach Anerkennung kann folglich als wichtiger Anreiz bei der Einhaltung humanitärer Normen dienen. Es lohnt also durchaus, Alternativen zu Dämonisierungs- und Kriminalisierungspraktiken zu identifizieren. Die internationale Gemeinschaft sollte den Dialog mit bewaffneten Gruppen suchen, wenn dadurch die Gewalt an Zivilisten gemindert und Wege zu Friedensverhandlungen eröffnet werden können.

\section{Literatur}

Allan, Pierre/Keller, Alexis 2012: Is a Just Peace Possible without Thin and Thick Recognition?, in: Erik Ringmar/Thomas Lindemann (eds): The International Politics of Recognition, Boulder, 71-84.

Bassiouni, CherifM. 2008: Criminal Law. The New Wars and the Crisis of Compliance with the Law of Armed Conflict by NonState Actors, in: The Journal of Criminal Law \& Criminology, 98: $3,711-810$.
Clapham, Andrew 2006: Human rights obligations of non-state actors in conflict situations, in: International Review of the Red Cross, 88: 863, 491-523.

Geneva Call 2005: Armed Non-State Actors and Landmines. Volume I: A Global Report Profiling NSAs and Their Use, Acquisition, Production, Transfer and Stockpiling of Landmines, Geneva.

Henckaerts, Jean-Marie 2003: Binding Armed Opposition Groups through Humanitarian Treaty Law and Customary Law, in: College of Europe (ed.): Relevance of International Humanitarian Law to Non-State Actors, Proceedings of the Bruges Colloquium, 25th-26th October 2002, No.27, 123-137.

Herr, Stefanie 2010: Vom Regelbruch zu politischer Verantwortung. Die Anerkennung völkerrechtlicher Normen durch nichtstaatliche Gewaltakteure, HSFK-Report Nr. 5/2010.

Internationales Komitee vom Roten Kreuz (IKRK) 2007: International Humanitarian Law and the Challenges of Contemporary Armed Conflict, in: www.icrc.org/Web/ eng/siteeng0.nsf/htmlall/5XRDCC/File/IHLcontemp_ armedconflicts_FINAL_ANG.pdf, 6.1.2010.

Johnson, Douglas H. 1998: The Sudan People's Liberation Army \& the Problem of Factionalism, in: Clapham, Christopher (ed.): African Guerillas, Oxford, 53-72.

Landmine Monitor 1999: Country Report Sudan, in: http:// lm.icbl.org/index.php/ publications/display?act=submit\&pqs_ year=1999\&pqs_type=lm\&pqs_report=sudan\&pqs_section=, 6.1.2010.

Landmine Monitor 2001: Country Report Sudan, in: http:// $1 \mathrm{~m}$.icbl.org/index.php/ publications/display?act=submit\&pqs_ year=2001\&pqs_type=lm\&pqs_report=sudan\&pqs_section=, 6.1.2010.

Lindemann, Thomas 2011: Peace Through Recognition: An Interactionist Interpretation of International Crisis, in: International Political Sociology 5:1, 68-86.

Meuser, Michael/Nagel, Ulrike 1991: ExpertInneninterviews vielfach erprobt, wenig bedacht: Ein Beitrag zur qualitativen Methodendiskussion, in: Garz, Detlef/Kraimer, Klaus (Hrsg.): Qualitativ-empirische Sozialforschung. Konzepte, Methoden, Analysen, Opladen, 441-470.

Murray, Michelle 2012: Recognition, Disrespect and the Struggle for Morocco. Rethinking Imperial Germany's Security Dilemma, in: Lindemann, Thomas/Ringmar, Erik (eds.): The International Politics of Recognition, Boulder, 131-151.

Riehl, Volker 2001: Who is Ruling in South Sudan? The Role of NGOs in Rebuilding Social Order, Studies on Emergencies and Disaster Relief No. 9, Copenhagen.

Risse, Thomas/Börzel, Tanja A. (i.E.): Human Rights in Areas of Limited Statehood. The New Agenda, in: Risse, Thomas/ Ropp, Stephen/Sikkink, Kathryn (eds.): From Commitment to Compliance. The Persistent Power of Human Rights, Cambridge.

Rolandsen, Oeystein H. 2005: Guerilla Government: Political Changes in the Southern Sudan during the 1990s, Copenhagen. 
Sassòli, Marco 2006: Transnational Armed Groups and International Humanitarian Law, Occasional Paper Series, Program on Humanitarian Policy and Conflict Research, Harvard University, Number 6.

Hofmann, Claudia/Schneckener, Ulrich 2010: Verhaltensänderung durch Normdiffusion? Die Ansätze von IKRK und Geneva Call im Umgang mit bewaffneten Gruppen, in: Die Friedens-Warte, 85, 4, 73-98. SPLM/A 2001: Statement of the Sudan People' Liberation Movement/Army on the Occasion of the Signing and Depositing to Geneva Call Deed of Commitment to Ban Landmines, 4. Oktober 2001, www.genevacall.org/resources/ nsas-statements/f-nsas-statements/2001-2010/2001-04octsplma.htm, 6.1.2010.

United Nations 2009: Honoring Geneva Conventions, Secretary-General Says Debate 'No Longer between Peace and Justice but between Peace and What Kind of Justice', UN Press Release SG/SM/12494, New York.

\title{
Hezbollah's Rise and Decline? How the Political Structure Seems to Harness the Power of Lebanon's Non-State Armed Group
}

\author{
Eva Dinge ${ }^{*}$
}

\begin{abstract}
In this article I will discuss the transformation from non-state armed group to governing party that the Lebanese Hezbollah has undergone since 2005. The 'hard facts' suggest a success story: the Shiite party has participated in various governments since 2005, brought down the cabinet in 2011, and controlled the formation of a new government. This has sparked widespread fears of an Islamist takeover of power in Lebanon. When we take a broader perspective, however, a different picture emerges: Hezbollah is more dependent on being part of the Lebanese political structure than its rhetoric would suggest or many observers assume. Participation in politics provides the group with much-needed legitimation as a democratic, civil actor, and shields it to some extent from international pressure to disarm. As long as it was enjoying full Syrian support and backing, Hezbollah has not hesitated to challenge the political status quo and risk political breakdown. However, since the beginning of the uprising in Syria, the party has had to tread more carefully. This explains why, since it brought down the Lebanese government in 2011, Hezbollah has largely played by the rules of the political game.
\end{abstract}

Keywords: Hezbollah, non-state armed group, Lebanon, Syria Hisbollah, nicht-staatliche bewaffnete Akteure, Libanon, Syrien

$\mathrm{E}$ ver since Hezbollah forced the resignation of Prime Minister Saad al-Hariri in January 2011 and was instrumental in influencing the make-up of the successor cabinet headed by Najib al-Mikati, the Islamist party-cum-militia is regarded as Lebanon's most powerful political player. As Hezbollah is a religious party and has embraced an agenda aimed at achieving social change, expectations that it would implement such changes after practically taking over power in Lebanon were high. This has been widely connected with fears of an Islamist takeover in Lebanon, the institution of religiously inspired policies, and generally a profound transformation of politics in Lebanon. At the time of writing, in January 2013, none of this has actually transpired. Instead, politics in Lebanon throughout the past two years, as tumultuous as it has been, has largely been "business as usual," in the sense that despite ongoing political conflict and crises, the nature of the political system has remained the same. Why have the expectations of change that Hezbollah's rise in political power had created not come true? I will argue that this is partly the result of the predominance

Eva Dingel is currently studying for a PhD at the Otto-Suhr-Institute at Free University Berlin. She is working on the topic of political strategies of Hezbollah and the Egyptian Muslim Brotherhood. of political structure over agency, and partly determined by the regional political situation and the party's implication in the assassination of former Prime Minister Rafik Hariri.

\section{Hezbollah's Hybrid Nature: Non-State Armed Group, Social Movement, Political Party}

Hezbollah, literally 'Party of God', was formed in Lebanon's Beqaa valley from 1982 onwards with Iranian support. Its formation was largely a response to Israel's invasion of South Lebanon the same year (for accounts of Hezbollah's foundation, see Aboul-Enein 2005; Alagha 2006; Hamzeh 2004; Norton 2009; Ollaik and Najjar 2012; Palmer Harik 2007; Qassem 2005; Saad-Ghorayeb 2002; Samaan 2007). The political domain and the military were intricately connected from the first days and months of Hezbollah's existence. The new organization also quickly set up various charities, which have bloomed into a comprehensive network of social-service providers (Deeb 2006; Hamzeh 2004, 52-3). When Lebanon's 15-year civil war (1975-1990) finally drew to an end, Hezbollah was the only wartime armed actor allowed to hold on to its weapons arsenal. All other militias were required to lay down 